\title{
The Genetics of Sudden Cardiac Death
}

\author{
Dan E. Arking ${ }^{1}$ and Nona Sotoodehnia ${ }^{2}$ \\ Dan E. Arking: arking@jhmi.edu; Nona Sotoodehnia: nsotoo@u.washington.edu \\ ${ }^{1}$ McKusick-Nathans Institute of Genetic Medicine, Johns Hopkins University School of Medicine, \\ Baltimore, Maryland 21209 \\ ${ }^{2}$ Division of Cardiology and Cardiovascular Health Research Unit, Department of Medicine, \\ University of Washington, Seattle, Washington 98101
}

\begin{abstract}
Sudden cardiac death (SCD), a sudden pulseless condition due to cardiac arrhythmia, remains a major public health problem despite recent progress in the treatment and prevention of overall coronary heart disease. In this review, we examine the evidence for genetic susceptibility to SCD in order to provide biological insight into the pathogenesis of this devastating disease and to explore the potential for genetics to impact clinical management of SCD risk. Both candidate gene approaches and unbiased genome-wide scans have identified novel biological pathways contributing to SCD risk. Although risk stratification in the general population remains an elusive goal, several studies point to the potential utility of these common genetic variants in high-risk individuals. Finally, we highlight novel methodological approaches to deciphering the molecular mechanisms involved in arrhythmogenesis. Although further epidemiological and clinical applications research is needed, it is increasingly clear that genetic approaches are yielding important insights into SCD that may impact the public health burden imposed by SCD and its associated outcomes.
\end{abstract}

\section{Keywords}

ventricular fibrillation; arrhythmia; electrophysiology; QT interval; QRS interval; association studies; GWAS

\section{INTRODUCTION}

In the United States, up to 450,000 adults die each year of sudden cardiac death (SCD), defined as a sudden and unexpected pulseless condition due to an arrhythmia without evidence of a noncardiac cause. SCD imposes a significant health burden, accounting for $10 \%-15 \%$ of total mortality and $40 \%-50 \%$ of mortality from coronary heart disease (CHD) $(35,36,49,90)$. Clinical and autopsy studies in Western populations have consistently demonstrated that the most common electrophysiological mechanism for SCD is ventricular fibrillation (VF) (see Figure 1) and that the most common pathological substrate is CHD $(45,54)$. It is important to note that despite advances in cardiopulmonary resuscitation, survival from SCD remains poor. Unfortunately, traditional CHD risk factors have a low predictive value for SCD, suggesting that other environmental or genetic factors are likely

Copyright $@ 2012$ by Annual Reviews.

DISCLOSURE STATEMENT

The authors are not aware of any affiliations, memberships, funding, or financial holdings that might be perceived as affecting the objectivity of this review. 
involved in the development of arrhythmias. Indeed, even among those with significant heart disease, it is difficult to identify those who will develop VF and SCD.

In this review, we examine genetic susceptibility to SCD and arrhythmogenesis in order to provide biological insight into SCD pathogenesis. We first focus on candidate gene studies that examine variation in genes involved in cardiac ventricular conduction and repolarization with the outcome of SCD in the general population. We then discuss unbiased genome-wide association studies (GWAS) of SCD. Finally, we detail novel approaches currently being undertaken to decipher the molecular mechanisms involved in arrhythmogenesis. Clearly, further epidemiological and clinical applications research on genetic susceptibility to VF and SCD is needed to reduce the public health burden imposed by SCD incidence and its associated outcomes.

\section{EVIDENCE OF GENETIC SUSCEPTIBILITY}

Population studies of SCD and familial studies of rare Mendelian arrhythmogenic disorders provide evidence for the heritability of myocardial vulnerability to VF and SCD. Population studies have demonstrated that a family history of SCD is a risk factor for developing SCD. A case-control study in Seattle and other parts of King County, Washington, showed that a parental history of SCD before age 65 was predictive of a 2.7 -fold increased risk of SCD, independent of parental history of myocardial infarction (47). Similar results were seen in the Paris Prospective Study, which showed that a parental history of SCD was associated with a 2-fold increase in the risk of SCD but not of myocardial infarction (56). If both parents had a history of SCD, there was a 9-fold increase in SCD risk.

Whereas the Seattle and Paris studies examined all out-of-hospital cardiac arrests that were due to SCD, two more recent studies examined familial aggregation specifically among individuals with SCD in the setting of acute ischemia. In a study of those with ST-elevation myocardial infarction in the Netherlands, a family history of SCD was more common among myocardial infarction cases with $\mathrm{VF}$ [odds ratio $(\mathrm{OR})=2.7$ ] than among myocardial infarction controls without VF (34). Another study, from Finland, showed that a history of SCD among first-degree relatives was more common among those with SCD than among those with nonfatal myocardial infarction $(\mathrm{OR}=1.6)$ or healthy controls $(\mathrm{OR}=2.2)(57)$. If a family history of SCD in two or more first-degree relatives was present, there was a marked increase in the odds of SCD compared with those with nonfatal myocardial infarction $(\mathrm{OR}=3.3)$ or healthy controls $(\mathrm{OR}=11.3)$.

Clearly, these studies demonstrate a familial aggregation of SCD, suggesting that shared genetic variation may influence vulnerability to VF and SCD. Importantly, that the effect of family history on SCD risk is independent of myocardial ischemia suggests that genetic factors may predispose to fatal arrhythmia specifically rather than to ischemic heart disease in general. It is noteworthy that despite varying population characteristics (the Netherlands and Finland studies included only SCD in the setting of acute ischemia, whereas the Seattle and Paris studies defined SCD more broadly), the findings across these four studies were consistent.

In addition to epidemiological studies demonstrating the heritability of SCD, remarkable progress has been made over the past two decades in identifying mutations that lead to rare inherited cardiac diseases associated with ventricular arrhythmias and SCD, such as long QT syndrome (LQTS), short QT syndrome (SQTS), Brugada syndrome, and catecholaminergic polymorphic ventricular tachycardia (CPVT), collectively defining a new group of syndromes, the inherited arrhythmogenic diseases (IADs) (59). These monogenic, primarily electrical disorders are predominantly, though not exclusively, caused by mutations in genes involved in the genesis, propagation, and repolarization of the electrical signal, and lead to 
altered electrocardiographic measures and morphology. The identified genes largely encode cardiac ion channel subunits, though genes involved in ion channel trafficking and calcium handling have also been implicated $(1,6,7,17,62,64)$. In addition to electrical disorders, inherited structural cardiomyopathies such as dilated cardiomyopathy, hypertrophic cardiomyopathy, and arrhythmogenic right ventricular cardiomyopathy can lead to altered electrophysiology and increase risk for $\operatorname{SCD}(30,53)$. Together, these rare Mendelian electrical and structural disorders lend further support to a genetic basis for SCD, and have allowed the identification of novel genes and pathways that influence the electrical system of the heart.

\section{ASSOCIATION OF GENETIC VARIANTS WITH SUDDEN CARDIAC DEATH}

Despite the progress of the past 20 years in the molecular characterization of IADs, much remains unknown about the genetic factors contributing to SCD risk in the general population. The methodologies for investigating common complex disorders are now well established, and new strategies are emerging. The allelic architecture of common complex diseases such as SCD is currently unknown, but is likely to involve both common variants with modest effects and an aggregation of rarer variants with stronger effects. Recent studies have examined the association of genetic variation with SCD using either a genome-wide approach, which focuses on common variants, or a candidate gene approach, which selects genes implicated in monogenic disorders or related to intermediate phenotypes that predispose to SCD, such as QT interval duration. Alternatively, an aggregation of rare variants in a gene may explain a proportion of the genetic risk for SCD in the population, and methods are being developed to examine the influence of rare variants. Table 1 summarizes the results described below.

\subsection{Candidate Gene Approach: Ion Channel Genes Associated with Arrhythmic Mendelian Disorders}

Before the GWAS era, and even still today, a substantial focus of SCD genetics was the role of genes implicated in rare Mendelian forms of disease that result in increased risk of sudden death. As noted above, a great deal of progress has been made on uncovering the genetics of these IADs (for an additional review, see 22), and a number of studies have reported associations between common variants in these genes and SCD risk in the general population.

The $S C N 5 A$ gene, which encodes the a subunit of the cardiac sodium channel responsible for cardiac action potential depolarization, has been one of the most extensively studied. Mutations in this gene have been associated with the LQT3 type of LQTS, Brugada syndrome, sick sinus syndrome, dilated cardiomyopathy, atrial fibrillation, and progressive cardiac conduction defect (77). Among African Americans, a common variant of the $S C N 5 A$ sodium channel gene $(S 1102 Y$, rs 7626962 , minor allele frequency $=0.068)$ that is absent in those of European or Asian descent has been associated with an eightfold increase in the risk of arrhythmias and syncope $[\mathrm{OR}=8.7,95 \%$ confidence interval $(\mathrm{CI})=3.2-23.9]$ (83). This variant accelerates sodium channel activation, leading the authors to hypothesize that in the setting of an additional risk factor, such as hypokalemia or structural heart disease, the risk of SCD would be increased for carriers of this allele. One limitation of this study is the heterogeneity of the cases, with varied phenotypes such as medication-induced prolonged QT interval, premature ventricular contractions, and infantile seizure all being combined into one case group, highlighting the need for replication in an independent study. The investigators also demonstrated that the $Y 1102$ allele was associated with a longer QT interval on the surface electrocardiogram (ECG) in a family-based study, although this finding has been disputed in a population-based study (55). Interestingly, the Y1102 allele was also shown to increase the risk of sudden infant death syndrome in a cohort of African 
Americans, and in vitro studies demonstrated a gain of function of these sodium channels in the presence of acidosis, which may occur during apneic sleep (72).

Burke et al. (19) subsequently examined the $S 1102 Y$ variant for SCD risk among AfricanAmerican autopsy subjects who had (a) a primary arrhythmic event without structural heart disease $(n=25),(b)$ SCD in the setting of mild to moderate left ventricular hypertrophy ( $n=$ $40)$, or (c) SCD with structural heart disease $(n=117)$. Comparing these subjects with a control group who died of noncardiac causes $(n=107)$, the investigators found that the Y1102 allele was more common in African Americans who died of unexpected arrhythmic causes $(\mathrm{OR}=8.4,95 \% \mathrm{CI}=2.1-28.6)$ or of $\mathrm{SCD}$ in the setting of mild ventricular hypertrophy $(\mathrm{OR}=4.9,95 \% \mathrm{CI}=1.3-13.4)$. Importantly, no association was found among those with structural heart disease (the largest case group) compared with controls. Hence, the findings are not fully conclusive as to whether $Y 1102$ increases the risk of SCD among African Americans, and further investigation is warranted.

Studies in European- and Asian-ancestry populations have provided equivocal results for the involvement of $S C N 5 A$ variants in common forms of SCD. Stecker et al. (84) examined genetic variation in the $S C N 5 A$ gene primarily among those of European descent by screening all 28 of the gene's coding exons for genetic variation among 67 SCD cases with known coronary artery disease (CAD) and $91 \mathrm{CAD}$ controls in the Oregon Sudden Unexpected Death Study, and found no association between coding variants and SCD risk. In contrast, Albert et al. (3) examined the Nurses' Health Study and the Health Professional Follow-Up Study for single-nucleotide polymorphisms (SNPs) in $S C N 5 A$ and four potassium channel genes (KCNQ1, KCNH2, KCNE1, and KCNE2). Five mutations or SNPs in $S C N 5 A$ were found in six women who died suddenly, a higher proportion than was seen in those who did not die suddenly. These same genes were examined for association with common variants in a nested case-control analysis of 516 cases and 1,522 matched controls of European ancestry (2). After accounting for multiple testing, two intronic SNPs remained significantly associated, one in $S C N 5 A$ and one in $K C N Q 1$, suggesting that both rare and common variants in $S C N 5 A$ may contribute to SCD risk. Clearly, these findings need to be replicated. Others have suggested that the A1673G variant in $S C N 5 A$ may modify risk in the Han Chinese $(24,44)$, whereas this and other SNPs have not been found in higher proportions in other populations with $\operatorname{SCD}(38,84)$.

In summary, although a number of studies have examined variation in IAD-associated genes as risk factors for SCD, with the most focus on $S C N 5 A$, none of these results have been convincingly validated in independent studies. Thus, although conceptually appealing, the relevance of genetic variants in IAD-associated genes to common forms of SCD remains to be demonstrated.

\subsection{Candidate Gene Approach: Genes Associated with Cardiac Ventricular Conduction (QRS) and Repolarization (QT)}

An alternative approach has been to focus on endophenotypes that are themselves risk factors for common forms of SCD, like the QRS and QT intervals, which are measured by a standard surface ECG (Figure 1). The QRS and QT interval durations-markers of cardiac ventricular conduction and repolarization, respectively_are heritable traits that are associated with SCD risk in healthy individuals as well as in individuals with cardiovascular-related diseases, such as hypertension and heart failure $(4,33,78,81,85)$. Because of the ready availability of large unselected populations with ECG data and because of the importance of QRS and QT interval perturbation (genetic or environmental) for arrhythmia risk, these endophenotypes may serve as useful and easily obtained intermediate phenotypes to identify genetic variation to be tested for SCD risk. The idea is that these 
genetic variants may themselves be risk factors for SCD or may at least identify genes that are risk factors.

Arking et al. (10) have successfully used this approach by focusing on identifying common genetic variants that modify the QT interval, which is under the joint control of the depolarizing $\mathrm{Na}^{+}$currents $\left(\mathrm{I}_{\mathrm{Na}}\right)$, the $\mathrm{Ca}^{2+}$ currents $\left(\mathrm{I}_{\mathrm{Ca}}\right)$, and the repolarizing $\mathrm{K}^{+}$currents ( $\mathrm{I}_{\mathrm{Ks}}$ and $\mathrm{I}_{\mathrm{Kr}}$ ). A number of studies have shown QT interval (corrected for heart rate) to be a moderately heritable trait (25\%-52\% heritability), indicating the likely involvement of genetic factors $(20,21,66)$. As noted above, extremes of QT interval have been associated with an increased risk for SCD in both Mendelian forms (LQTS and SQTS) and populationbased settings $(31-33,43,69,78,80)$. These observations motivated one of the first successful GWAS, in which the investigators sampled from the extremes of the QT interval distribution in a population-based sample of European ancestry and looked for common variants associated with the QT interval (10). A common variant in the $5^{\prime}$ region of the NOS1AP gene was associated with a 2-3-ms increase in QT interval per minor allele. NOS1AP encodes an adapter protein that serves to physically bridge neuronal nitric oxide synthase with its targets and modulator proteins. In guinea pig ventricular myocytes, NOS1AP is localized near ryanodine receptors, and its over-expression results in a shortening of the cardiac action potential, a decrease in the L-type $\mathrm{Ca}^{2+}\left(\mathrm{I}_{\mathrm{Ca}}\right)$ current, and a smaller increase in the rapid delayed rectifier $\mathrm{K}^{+}$current $\left(\mathrm{I}_{\mathrm{Kr}}\right)$, resulting in a shortening of the QT interval (23). The genetic association has been replicated widely in populations of European ancestry $(40,60,73,75,86)$ and more recently in an African-American population (9).

To test the underlying hypothesis that genes associated with QT interval may also modify SCD risk, Kao et al. (58) genotyped a series of SNPs in the NOS1APlocus to look for association with SCD in the combined Atherosclerosis Risk in Communities Study and Cardiovascular Health Study cohorts (498 cases, 19,295 controls). The SNP most strongly associated with QT interval, rs16847548, was also associated with risk for SCD in white US adults, with the QT-prolonging variant (C allele) associated with increased SCD risk ( $p=$ 0.002). Individuals carrying the CC genotype at rs 16847548 of NOS1AP were approximately $72 \%$ more likely to die of SCD and had a mean QT interval that was approximately $5 \mathrm{~ms}$ longer than that of their counterparts with the TT genotype, even after accounting for age, sex, and heart rate, with the genetic effect specific to SCD as opposed to non-SCD cardiovascular mortality or overall mortality. The at-risk $\mathrm{C}$ allele is common, with $39 \%$ of the general white population carrying one copy of the $\mathrm{C}$ allele and $5 \%$ carrying two copies. Further characterization of this genomic region identified a downstream neighboring SNP, rs12567209, that was not correlated with rs16847548, which was also independently associated with SCD in whites. In contrast to rs16847548, the minor allele displayed a protective effect, with carriers ( $14 \%$ of white individuals) having a $53 \%$ reduction in the risk for SCD $(p=0.003)$. Adjustment for QT interval and CHD risk factors attenuated but did not eliminate the association between rs16847548 and SCD, and such adjustment had no effect on the association between rs12567209 and SCD. A second independent study by Eijgelsheim et al. (41) confirmed these findings.

Following the identification of additional QT interval-associated loci through larger GWAS $(65,70)$, Noseworthy et al. (68) explored the relationship between SCD and common genetic variants that influence the QT interval in a Finnish population-based study. Although they strongly replicated the association between common variants and QT interval and between QT interval and SCD, a QT genetic risk score did not show a significant linear relationship with SCD risk, with only the top two quintiles associated with increased risk for SCD. The authors noted that a lack of association may be due to limited power (only 116 incident SCDs were available) or may mean that not all QT-prolonging alleles actually 
increase SCD risk. This latter point is particularly relevant in light of recent analyses demonstrating that some variants that prolong the electrocardiographic phenotype may actually decrease arrhythmia risk. This has been shown for PR genetic variants and association with atrial fibrillation as well as QRS variants and risk for SCD (see below) (71, 82). Thus, constructing a genetic risk score may be more challenging, and will likely need to incorporate information from across the ECG measures that influence SCD. Nevertheless, a properly constructed risk score is likely to be useful for several reasons. First, it may serve as a measure of lifetime exposure to a prolonged QT interval, as opposed to a single measure taken in a clinical setting. Second, as Noseworthy et al. (68) point out, the genetic score may measure risk not captured in an ECG. Specifically, repolarization may remain normal in an individual with a variant owing to redundant mechanisms, but the "repolarization reserve" (76) may be reduced and unmasked by exposure to drugs or ischemia, resulting in increased risk for SCD.

A more comprehensive assessment of the role of ECG-associated SNPs in SCD risk was recently conducted as part of a GWAS for SCD (described in more detail below). Arking et al. (8) examined 49 SNPs that had been previously associated $\left(p<5 \times 10^{-8}\right)$ with QRS, QT, and RR (inverse heart rate) intervals in an SCD GWAS composed of 1,283 SCD cases and $>20,000$ controls. In aggregate, the ECG-trait-prolonging allele was more often associated with increased risk for SCD (31 of 49, $p=0.03$ ), though this effect was entirely driven by the QRS/QT-associated SNPs (28 of 40, $p=0.006$ ). Nominal association was observed for three loci ( $P L N, K C N Q 1$, and NOS1AP), and a fourth locus (TKT/CACNA1D/PRKCD) was significant even after Bonferroni correction for the number of loci tested. The TKT/ $C A C N A 1 D / P R K C D$ locus is particularly interesting, as the QRS-prolonging allele was protective for risk of SCD, suggesting that the increased SCD risk associated with this allele is not necessarily mediated through its effect on increasing QRS interval.

These endophenotype-based candidate gene approaches highlight several important points. First, focusing on broadly measured traits that are themselves risk factors for SCD can minimize the number of loci examined to uncover genetic variants that may not be found by an unbiased genome-wide approach owing to limited power. Second, by and large, common coding variants do not explain the observed effects, highlighting the likely role of regulatory variants $(10,42,65,70,71,82)$. Third, as exemplified by the TKT/CACNA1D/PRKCD locus, although a gene is identified through its association with an endophenotype, the direction of effect need not follow the expected pattern (i.e., increasing QRS/QT interval with increased SCD risk), suggesting that the mechanism of action need not necessarily be mediated through the effect on the endophenotype. This was also observed for NOS1AP, where one of the alleles that was associated with SCD had no effect on QT interval (58). Finally, we note that although screening for SCD-associated genes using an endophenotype can be successful, it is in no way comprehensive for the identification of these genes, highlighting the importance of also conducting a large-scale GWAS directly for SCD.

\subsection{Genome-Wide Association Studies for Sudden Cardiac Death}

There have been several GWAS for SCD, though only two have reported genome-wide significant results. The first such study was conducted in the AGNES (A in the Netherlands) cohort, which focuses on a highly specific population of individuals with a first myocardial infarction and VF who survived to hospital admission $(n=515)$ compared with individuals with myocardial infarction alone $(n=457)$, with replication in 146 out-ofhospital SCD cases and 391 controls (15). Only one SNP achieved genome-wide significance: rs 2824292 in the $21 \mathrm{q} 21$ locus, with an OR of 1.78 (95\% $\mathrm{CI}=1.47-2.13, p=$ $\left.3.36 \times 10^{-10}\right)$ in the initial study and an OR of $1.49(95 \% \mathrm{CI}=1.14-1.95, p=0.004)$ in the replication samples alone. This is a common variant (allele frequency of 47\%) that is in an intergenic region, and the closest gene, $C X A D R(\sim 98 \mathrm{~kb}$ away), encodes a viral receptor 
previously implicated in viral myocarditis (18). Somewhat surprisingly, this SNP association was not replicated in a larger GWAS (described below). This lack of replication may reflect an association specific to the populations studied, or perhaps one restricted to those with a first myocardial infarction who survived a VF event as opposed to a more broadly defined class of SCD in the general population.

The second GWAS utilized a total of 4,400 SCD cases and >30,000 controls, all of European ancestry, in a two-stage study design (8). In the first stage, 1,283 SCD cases and 20,000 controls were scanned genome-wide, with validation of suggestive findings conducted in 3,119 SCD cases and >11,000 controls. In a combined analysis of GWAS and all replication studies, the most significant result was seen at the $2 \mathrm{q} 24.2$ locus, with the strongest signal, rs4665058 $\left(p=1.8 \times 10^{-10}\right)$, mapped to an intron of $B A Z 2 B$. This locus contains three genes not previously known to play a role in cardiac biology $(B A Z 2 B$, WDSUB1, and TANC1), though all are expressed in the heart (http://www.genecards.org). The risk allele (A allele) of rs 4665058 had a study-size-weighted frequency of $1.4 \%$ and increased the risk for SCD by 1.92 -fold per allele $(95 \% \mathrm{CI}=1.57-2.34)$ in the combined analysis. Interestingly, the risk allele is the ancestral allele (based on nonhuman primate sequence), and its frequency in European-ancestry populations suggests strong negative selection, as fewer than $0.8 \%$ of ancestral alleles have reached a frequency of $1.4 \%$ or lower. Based on pilot 1 data from the 1000 Genomes Project (November 2010 release) (39), no missense/splice mutations were highly correlated with rs4665058 $\left(r^{2}>0.8\right)$, suggesting that the functional variant is likely regulatory. It is interesting to note that the meta-analysis consisted of both population-based and case-control studies, with some of the case-control studies using CAD controls as opposed to population-based controls. The consistent results in studies with both CAD controls and population-based controls (see supplementary figure 2 from Reference 8) provide evidence that the risk associated with rs 4665058 may be specific to SCD rather than a generic CAD risk factor. Indeed, given the case mix across the cohorts, the study was best powered to identify variants that increase the risk for SCD through a mechanism common across the various subtypes of SCD.

\section{FUTURE DIRECTIONS: OPPORTUNITIES AND CHALLENGES}

The challenge of the post-human-genome era is to uncover the molecular mechanisms for complex diseases such as SCD in order to better understand the biology of the disease, develop novel therapies, identify those at higher risk, and deliver on the promise of personalized medicine. This last point is of particular relevance to SCD; one of the most common adverse drug reactions for which medications are removed from the market is prolongation of the QT interval and potential for SCD. Advances in genomic technologies have enabled the efficient and comprehensive interrogation of the entire genome for proarrhythmic variants. With large sample sizes (thousands of cases and thousands of controls), genomic risk factors contributing to SCD can be identified. A number of challenges remain, and here we discuss the underlying homogeneity of this complex disease, interactions with the genetic and environmental milieu, and novel interrogation of the genome.

\subsection{Phenotype}

SCD is a complex phenotype that often occurs during the interplay of two processes: (a) a transient triggering event, such as ischemia or adrenergic stress, that occurs in the setting of (b) a susceptible proarrhythmic myocardial substrate. The predominant myocardial pathology in Western populations is CHD, with or without prior myocardial infarction or heart failure, though a variety of other, less common subphenotypes exist, such as those with cardiac hypertrophy or valvular pathology and those with structurally normal hearts that have a channelopathy leading to arrhythmogenic vulnerability. 
One hypothesis is that SCD due to VF is a final common event representing myocardial electrical instability, regardless of the underlying myocardial substrate, and therefore that genetic variants exist that increase SCD risk among a variety of cardiac disease substrates for VF. Although the magnitude of this risk may differ across substrates, the direction of the association will likely be similar. Further investigation is needed, but several lines of evidence support this hypothesis. First, population studies of SCD have been able to identify variation associated with SCD through meta-analyses of the findings of studies with differing designs, case ascertainment, and operational definitions used to classify SCD. Furthermore, the identified genetic factors, such as those in NOS1AP, influence SCD risk in the general population as well as in select subgroups with a high risk of SCD that is not dependent on CAD status, such as those with Mendelian forms of LQTS (see below).

The often-used Hinkle-Thaler and World Health Organization systems for classifying mode of death define SCD as a sudden, unexpected pulseless condition that occurs within an hour of acute cardiac symptoms, during sleep, or within 24 hours of last being seen alive and well $(16,26,46,50,52)$. Given the operational challenges of identifying those with SCD, a number of varying SCD criteria have appeared in the literature. Furthermore, the cardiac rhythm at clinical presentation is often unknown. Despite this potential heterogeneity in case definition, the results of prior epidemiological studies examining determinants of SCD have typically been consistent. Nonetheless, phenotypic homogeneity is important to maximize the opportunities to identify genetic loci. Information on rhythm at time of arrest obtained from paramedic records, information on underlying heart pathology obtained from autopsy and prior medical records, and information on environmental factors such as medication use will assist with homogenizing the phenotype.

\subsection{Genetic and Environmental Milieu}

Genes do not act in isolation, but rather exert their effects in the context of the genetic and environmental milieu. Complex allele-allele interactions are an emerging and important area of investigation in GWAS. It is clear that even in monogenic IADs, family members who share the same mutation can have different phenotypic expressions of the disease. For instance, QT intervals vary between different afflicted members of the same LQTS family. This variance may be due to low penetrance (74) but may also be influenced by compound mutations or common SNPs $(28,79,89)$. Tomas et al. (87) examined this hypothesis by exploring the role of NOS1AP variants in patients with Mendelian forms of LQTS (LQT13 ). The overall risk of a severe cardiac event was $22 \%$, and the NOS1AP genotype increased risk by 1.63 -fold $(95 \% \mathrm{CI}=1.06-2.50)$ irrespective of the specific underlying LQTS mutation. Interestingly, this genetic effect was independent of the measured QT interval. Similar findings were reported by Crotti et al. (29), who examined NOS1APvariants in LQTS patients with a single founder mutation in $K C N Q 1$. Approximately $25 \%$ of mutation carriers experienced cardiac arrest and/or SCD, and a 1.8-fold increased risk $(95 \% \mathrm{CI}=1.1-$ 3.3) was observed for carriers of the minor NOS1AP allele. Examining the $3^{\prime}$ untranslated region of $K C N Q 1$ in patients with LQT1, which is caused by heterozygous Mendelian mutations in KCNQ1, Amin et al. (5) demonstrated that 3' untranslated-region SNPs modify disease severity, with the effects dependent on whether the derived allele is in cis or trans to the mutant allele. This result is explained by luciferase reporter assays showing that the derived allele reduces gene expression, so that when in cis, it results in lower mutant protein levels (lowering QT interval), whereas in trans, it results in lower wild-type protein levels and exacerbates the QT prolongation. Thus, in addition to simply genotyping high-risk individuals, examining genetic context is extremely important. Indeed, given the high rates of severe cardiac events in these populations and the high cost of intervention-implantable cardiodefibrillators (ICDs) cost more than $\$ 40,000$ per patient—being able to identify those at high and, equally important, low risk has important clinical implications. 
Environmental factors clearly influence both SCD risk and its intermediate phenotypes. For instance, one of the most common reasons for the market withdrawal of a medication is its effect on the QT interval and its potential for arrhythmogenesis. A number of cardiac and noncardiac medications interact with the slow delayed rectifying potassium channel, part of the voltage-gated ion channel complex responsible for repolarization, which may lead to QT prolongation and proarrhythmia. Genetic variation in ion channel genes involved in repolarization have been associated with acquired LQTS, suggesting that variants interact with certain medications to increase the risk of QT prolongation and arrhythmias. Detailed medication data will be necessary to explore important gene-environment interactions.

\subsection{Novel Analytic Approaches}

Although there is no doubt that more refined phenotyping and increased sample size are of critical importance in identifying genetic variants associated with SCD, there are a number of computational approaches and active methodology developments that will increase statistical power. A detailed discussion of improvements to GWAS methods is beyond the scope of this review, though we point out that given the vast amounts of exome and wholegenome sequence data becoming available, there is currently a great deal of interest in combining tests for rare and common variants. Most current analytic strategies involve running a standard common variant test (regression) for SNPs with a minor allele frequency of $\geq 1 \%$ and then separately running a burden test (for reviews, see 12-14, 61) for rare variants. The threshold for calling something a rare variant is unclear, and there is likely to be overlap between the common and rare variant tests owing to linkage disequilibrium. Putting both of these tests into a unified statistical framework is likely to increase power. The GWAS approach is also likely to be improved through the incorporation of knowledge of biological pathways and functional annotation of the genome.

In addition to improving the methods, incorporating additional genome-scale data is likely to impact our ability to identify SCD-associated genes. Indeed, there is emerging evidence that combining gene expression data with genetic variation is a powerful tool to identify genes underlying disease (for review, see 27). Many of these studies have focused on a role for expression in interpreting GWAS results, using eSNPs (SNPs that alter gene expression) to identify the likely gene in a region of association containing multiple genes. More recently, studies incorporating both GWAS and expression data into their primary study design have emerged, with a recent study of late-onset Alzheimer's disease incorporating expression data from both affected and control individuals (88). Thus, beyond single-locus eQTL (expression quantitative trait locus) mapping, the combined use of genetic variation and altered gene expression between cases and controls is becoming a powerful tool to elucidate molecular networks in disease. A seminal example by Chen et al. (25) used a mouse model to identify a macrophage-enriched network that has a causal relationship with disease traits associated with metabolic syndrome. Indeed, in the absence of known genes in a pathway, altered gene expression between cases and controls can be a powerful tool in network analysis and can readily be combined with association study results to identify diseasecausing genes (67).

Although no one has yet combined GWAS and expression data in the context of a primary screen for genetic dissection of cardiovascular disease, a number of studies have looked for altered gene expression in the context of diseased hearts, including those with chronic atrial fibrillation, dilated cardiomyopathy, and several right ventricular arrhythmogenic diseases. Transcriptional profiling of 77 ion channel genes clearly distinguished between Brugada syndrome and control subjects using hierarchical clustering (48). Moreover, the Brugada patients were distinguishable from those with right ventricular tachycardia due to other causes (arrhythmogenic right ventricular cardiomyopathy and idiopathic right ventricular outflow-tract tachycardia). These data suggest that transcriptome profiling may aid in 
elucidating the etiology of SCD in three ways. First, incorporating differential expression between cases and controls will add to the statistical power for GWAS (see Figure 2). Second, eQTL mapping may aid in pinpointing the genetic effect in an associated region; it is important to note that $\sim 70 \%$ of eQTLs are tissue specific $(37,51)$, highlighting the importance of collecting cardiac tissue. Finally, transcriptome profiling may allow for further classification of SCD patients, which would reduce genetic heterogeneity. This combined approach, marrying GWAS and expression data, is likely to serve as a framework for incorporating additional layers of data from protein expression, methylation, and other techniques.

\section{CONCLUSION}

There are three main areas in which understanding the genetics of SCD will potentially impact our understanding of, and response to, SCD risk. First, gene identification will likely reveal novel biological pathways (e.g., NOS1AP for QT interval) that will help to elucidate the etiologies of SCD. Although some genes are likely to influence the risk for all forms of $\mathrm{SCD}$, it is also possible that specific genes will influence the risk for specific forms of SCD. Indeed, understanding the different causes of SCD will likely require the comprehensive phenotyping currently generated through a number of autopsy-based SCD studies, allowing investigators to pathologically, genetically, and molecularly dissect the different etiologies of SCD. The second impact will be to identify a series of candidate genes that will serve as potential therapeutic targets for those at high risk for SCD (e.g., LQTS patients). These candidate genes may also serve as potential biomarkers to identify individuals at high risk. Although it is unlikely that the genetic risk profile alone will be sufficient to classify otherwise asymptomatic individuals as high risk, given that GWAS typically identify variants that explain $<10 \%$ of the overall variance (63), it is possible that some of the proteins from the associated genes may be altered as a shared final event in common forms of SCD. This scenario has been observed in arrhythmogenic right ventricular cardiomyopathy, a rare but strongly genetic form of heart disease that leads to SCD. Despite considerable locus heterogeneity, with causative mutations in five separate genes, $>90 \%$ of individuals with this disease share a final common event of decreased plakoglobin protein expression at myocyte junctions (11). This observation suggests that despite the large number of genetic polymorphisms, a single protein or a limited number of proteins may be commonly altered in individuals susceptible to SCD. Finally, although genetic profiling may be of marginal utility in the general population, it may have a much greater value in highrisk populations such as those with Mendelian disorders (such as LQTS, SQTS, and Brugada syndrome) and/or candidates for primary prophylaxis with ICDs.

Genomic approaches, even at this early stage, have already yielded important new insights into SCD risk in both the general population and high-risk groups. Given the significant levels of overall mortality due to SCD, and with interventions limited to beta-blockade usage and ICDs, there is an ongoing and urgent need to identify additional therapeutic targets. This urgency is further exacerbated by the inability to identify those requiring intervention and, perhaps of equal importance, those who do not require an invasive and high-cost intervention such as an ICD. Although further work is required to translate these and additional findings to the clinic, there is now a strong foundation for the ultimate goal of harnessing the power of human genetics to lessen the significant public health burden imposed by SCD.

\section{Acknowledgments}

We thank Dr. Marc Halushka and Dr. Jordan Prutkin for helpful comments. This work is supported by the National Heart Lung and Blood Institute (1R01HL111267 to D.E.A. and R01 HL088456 to N.S.). 


\section{LITERATURE CITED}

1. Ackerman MJ. Genotype-phenotype relationships in congenital long QT syndrome. J Electrocardiol. 2005; 38:64-68. [PubMed: 16226077]

2. Albert CM, MacRae CA, Chasman DI, VanDenburgh M, Buring JE, et al. Common variants in cardiac ion channel genes are associated with sudden cardiac death. Circ Arrhythm Electrophysiol. 2010; 3:222-29. [PubMed: 20400777]

3. Albert CM, Nam EG, Rimm EB, Jin HW, Hajjar RJ, et al. Cardiac sodium channel gene variants and sudden cardiac death in women. Circulation. 2008; 117:16-23. [PubMed: 18071069]

4. Algra A, Tijssen JG, Roelandt JR, Pool J, Lubsen J. QTc prolongation measured by standard 12-lead electrocardiography is an independent risk factor for sudden death due to cardiac arrest. Circulation. 1991; 83:1888-94. [PubMed: 2040041]

5. Amin AS, Giudicessi JR, Tijsen AJ, Spanjaart AM, Reckman YJ, et al. Variants in the 3' untranslated region of the KCNQ1-encoded Kv7.1 potassium channel modify disease severity in patients with type 1 long QT syndrome in an allele-specific manner. Eur Heart J. 2012; 33:714-23. [PubMed: 22199116]

6. Antzelevitch C. Brugada syndrome. Pacing Clin Electrophysiol. 2006; 29:1130-59. [PubMed: 17038146]

7. Antzelevitch C, Pollevick GD, Cordeiro JM, Casis O, Sanguinetti MC, et al. Loss-of-function mutations in the cardiac calcium channel underlie a new clinical entity characterized by ST-segment elevation, short QT intervals, and sudden cardiac death. Circulation. 2007; 115:442-49. [PubMed: 17224476]

8. Arking DE, Junttila MJ, Goyette P, Huertas-Vazquez A, Eijgelsheim M, et al. Identification of a sudden cardiac death susceptibility locus at 2q24.2 through genome-wide association in European ancestry individuals. PLoS Genet. 2011; 7:e1002158. [PubMed: 21738491]

9. Arking DE, Khera A, Xing C, Kao WH, Post W, et al. Multiple independent genetic factors at NOS1AP modulate the QT interval in a multi-ethnic population. PLoS ONE. 2009; 4:e4333. [PubMed: 19180230]

10. Arking DE, Pfeufer A, Post W, Kao WH, Newton-Cheh C, et al. A common genetic variant in the NOS1 regulator NOS1AP modulates cardiac repolarization. Nat Genet. 2006; 38:644-51. [PubMed: 16648850]

11. Asimaki A, Tandri H, Huang H, Halushka MK, Gautam S, et al. A new diagnostic test for arrhythmogenic right ventricular cardiomyopathy. N Engl J Med. 2009; 360:1075-84. [PubMed: 19279339]

12. Asimit J, Zeggini E. Rare variant association analysis methods for complex traits. Annu Rev Genet. 2010; 44:293-308. [PubMed: 21047260]

13. Bansal V, Libiger O, Torkamani A, Schork NJ. Statistical analysis strategies for association studies involving rare variants. Nat Rev Genet. 2010; 11:773-85. [PubMed: 20940738]

14. Basu S, Pan W. Comparison of statistical tests for disease association with rare variants. Genet Epidemiol. 2011; 35:606-19. [PubMed: 21769936]

15. Bezzina CR, Pazoki R, Bardai A, Marsman RF, de Jong JS, et al. Genome-wide association study identifies a susceptibility locus at 21q21 for ventricular fibrillation in acute myocardial infarction. Nat Genet. 2010; 42:688-91. [PubMed: 20622880]

16. Bigger JT Jr, Whang W, Rottman JN, Kleiger RE, Gottlieb CD, et al. Mechanisms of death in the CABG Patch trial: a randomized trial of implantable cardiac defibrillator prophylaxis in patients at high risk of death after coronary artery bypass graft surgery. Circulation. 1999; 99:1416-21. [PubMed: 10086963]

17. Bjerregaard P, Gussak I. Short QT syndrome. Ann Noninvasive Electrocardiol. 2005; 10:436-40. [PubMed: 16255754]

18. Bowles NE, Richardson PJ, Olsen EG, Archard LC. Detection of Coxsackie-B-virus-specific RNA sequences in myocardial biopsy samples from patients with myocarditis and dilated cardiomyopathy. Lancet. 1986; 1:1120-23. [PubMed: 2871380]

19. Burke A, Creighton W, Mont E, Li L, Hogan S, et al. Role of SCN5A Y1102 polymorphism in sudden cardiac death in blacks. Circulation. 2005; 112:798-802. [PubMed: 16061744] 
20. Busjahn A, Knoblauch H, Faulhaber HD, Boeckel T, Rosenthal M, et al. QT interval is linked to 2 long-QT syndrome loci in normal subjects. Circulation. 1999; 99:3161-64. [PubMed: 10377080]

21. Carter N, Snieder H, Jeffery S, Saumarez R, Varma C, et al. QT interval in twins. J Hum Hypertens. 2000; 14:389-90. [PubMed: 10878701]

22. Cerrone M, Priori SG. Genetics of sudden death: focus on inherited channelopathies. Eur Heart J. 2011; 32:2109-18. [PubMed: 21478491]

23. Chang KC, Barth AS, Sasano T, Kizana E, Kashiwakura Y, et al. CAPON modulates cardiac repolarization via neuronal nitric oxide synthase signaling in the heart. Proc Natl Acad Sci USA. 2008; 105:4477-82. [PubMed: 18337493]

24. Chen J, Xie X, Zhu J, Tao Q, Wang X. Single-nucleotide polymorphisms in SCN5A gene in Chinese Han population and their correlation with cardiac arrhythmias. Genet Med. 2004; 6:159. [PubMed: 15354335]

25. Chen Y, Zhu J, Lum PY, Yang X, Pinto S, et al. Variations in DNA elucidate molecular networks that cause disease. Nature. 2008; 452:429-35. [PubMed: 18344982]

26. Chugh SS, Jui J, Gunson K, Stecker EC, John BT, et al. Current burden of sudden cardiac death: multiple source surveillance versus retrospective death certificate-based review in a large US community. J Am Coll Cardiol. 2004; 44:1268-75. [PubMed: 15364331]

27. Cookson W, Liang L, Abecasis G, Moffatt M, Lathrop M. Mapping complex disease traits with global gene expression. Nat Rev Genet. 2009; 10:184-94. [PubMed: 19223927]

28. Crotti L, Lundquist AL, Insolia R, Pedrazzini M, Ferrandi C, et al. KCNH2-K897T is a genetic modifier of latent congenital long-QT syndrome. Circulation. 2005; 112:1251-58. [PubMed: 16116052]

29. Crotti L, Monti MC, Insolia R, Peljto A, Goosen A, et al. NOS1AP is a genetic modifier of the long-QT syndrome. Circulation. 2009; 120:1657-63. [PubMed: 19822806]

30. Dalal D, Nasir K, Bomma C, Prakasa K, Tandri H, et al. Arrhythmogenic right ventricular dysplasia: a United States experience. Circulation. 2005; 112:3823-32. [PubMed: 16344387]

31. de Bruyne MC, Hoes AW, Kors JA, Hofman A, van Bemmel JH, Grobbee DE. Prolonged QT interval predicts cardiac and all-cause mortality in the elderly: the Rotterdam Study. Eur Heart J. 1999; 20:278-84. [PubMed: 10099922]

32. Dekker JM, Crow RS, Hannan PJ, Schouten EG, Folsom AR. Heart rate-corrected QT interval prolongation predicts risk of coronary heart disease in black and white middle-aged men and women: the ARIC study. J Am Coll Cardiol. 2004; 43:565-71. [PubMed: 14975464]

33. Dekker JM, Schouten EG, Klootwijk P, Pool J, Kromhout D. Association between QT interval and coronary heart disease in middle-aged and elderly men. The Zutphen Study. Circulation. 1994; 90:779-85. [PubMed: 8044948]

34. Dekker LR, Bezzina CR, Henriques JP, Tanck MW, Koch KT, et al. Familial sudden death is an important risk factor for primary ventricular fibrillation: a case-control study in acute myocardial infarction patients. Circulation. 2006; 114:1140-45. [PubMed: 16940195]

35. Demirovic J, Myerburg RJ. Epidemiology of sudden coronary death: an overview. Prog Cardiovasc Dis. 1994; 37:39-48. [PubMed: 8022985]

36. de Vreede-Swagemakers JJ, Gorgels AP, Dubois-Arbouw WI, van Ree JW, Daemen MJ, et al. Out-of-hospital cardiac arrest in the 1990's: a population-based study in the Maastricht area on incidence, characteristics and survival. J Am Coll Cardiol. 1997; 30:1500-5. [PubMed: 9362408]

37. Dimas AS, Deutsch S, Stranger BE, Montgomery SB, Borel C, et al. Common regulatory variation impacts gene expression in a cell type-dependent manner. Science. 2009; 325:1246-50. [PubMed: 19644074]

38. Doolan A, Langlois N, Chiu C, Ingles J, Lind JM, Semsarian C. Postmortem molecular analysis of KCNQ1 and SCN5A genes in sudden unexplained death in young Australians. Int J Cardiol. 2008; 127:138-41. [PubMed: 17544529]

39. Durbin RM, Abecasis GR, Altshuler DL, Auton A, Brooks LD, et al. A map of human genome variation from population-scale sequencing. Nature. 2010; 467:1061-73. [PubMed: 20981092]

40. Eijgelsheim M, Aarnoudse AL, Rivadeneira F, Kors JA, Witteman JC, et al. Identification of a common variant at the NOS1APlocus strongly associated to QT-interval duration. Hum Mol Genet. 2008; 18:347-57. [PubMed: 18927126] 
41. Eijgelsheim M, Newton-Cheh C, Aarnoudse AL, van Noord C, Witteman JC, et al. Genetic variation in NOS1AP is associated with sudden cardiac death: evidence from the Rotterdam Study. Hum Mol Genet. 2009; 18:4213-18. [PubMed: 19643915]

42. Eijgelsheim M, Newton-Cheh C, Sotoodehnia N, de Bakker PI, Muller M, et al. Genome-wide association analysis identifies multiple loci related to resting heart rate. Hum Mol Genet. 2010; 19:3885-94. [PubMed: 20639392]

43. Elming H, Holm E, Jun L, Torp-Pedersen C, Kober L, et al. The prognostic value of the QT interval and QT interval dispersion in all-cause and cardiac mortality and morbidity in a population of Danish citizens. Eur Heart J. 1998; 19:1391-400. [PubMed: 9792266]

44. Fang DH, Wu LQ, Lu L, Lou S, Gu G, et al. Association of human SCN5A polymorphisms with idiopathic ventricular arrhythmia in a Chinese Han cohort. Circ J. 2008; 72:592-97. [PubMed: 18362431]

45. Farb A, Tang AL, Burke AP, Sessums L, Liang Y, Virmani R. Sudden coronary death: frequency of active coronary lesions, inactive coronary lesions, and myocardial infarction. Circulation. 1995; 92:1701-9. [PubMed: 7671351]

46. Fisher JD, Buxton AE, Lee KL, Packer DL, Echt DS, et al. Designation and distribution of events in the Multicenter UnSustained Tachycardia Trial (MUSTT). Am J Cardiol. 2007; 100:76-83. [PubMed: 17599445]

47. Friedlander Y, Siscovick DS, Arbogast P, Psaty BM, Weinmann S, et al. Sudden death and myocardial infarction in first degree relatives as predictors of primary cardiac arrest. Atherosclerosis. 2002; 162:211-16. [PubMed: 11947916]

48. Gaborit N, Wichter T, Varro A, Szuts V, Lamirault G, et al. Transcriptional profiling of ion channel genes in Brugada syndrome and other right ventricular arrhythmogenic diseases. Eur Heart J. 2009; 30:487-96. [PubMed: 19029124]

49. Gillum RF. Sudden coronary death in the United States: 1980-1985. Circulation. 1989; 79:756-65. [PubMed: 2924409]

50. Greenberg H, Case RB, Moss AJ, Brown MW, Carroll ER, Andrews ML. Analysis of mortality events in the Multicenter Automatic Defibrillator Implantation Trial (MADIT-II). J Am Coll Cardiol. 2004; 43:1459-65. [PubMed: 15093884]

51. Heinzen EL, Ge D, Cronin KD, Maia JM, Shianna KV, et al. Tissue-specific genetic control of splicing: implications for the study of complex traits. PLoS Biol. 2008; 6:e1. [PubMed: 19222302]

52. Hinkle LE Jr, Thaler HT. Clinical classification of cardiac deaths. Circulation. 1982; 65:457-64. [PubMed: 7055867]

53. Ho CY, Seidman CE. A contemporary approach to hypertrophic cardiomyopathy. Circulation. 2006; 113:e858-62. [PubMed: 16785342]

54. Holmberg M, Holmberg S, Herlitz J. An alternative estimate of the disappearance rate of ventricular fibrillation in out-of-hospital cardiac arrest in Sweden. Resuscitation. 2001; 49:219-20. [PubMed: 11428360]

55. Jeff JM, Brown-Gentry K, Buxbaum SG, Sarpong DF, Taylor HA, et al. SCN5A variation is associated with electrocardiographic traits in the Jackson Heart Study. Circ Cardiovasc Genet. 2011; 4:139-44. [PubMed: 21325150]

56. Jouven X, Desnos M, Guerot C, Ducimetiere P. Predicting sudden death in the population: the Paris Prospective Study I. Circulation. 1999; 99:1978-83. [PubMed: 10209001]

57. Kaikkonen KS, Kortelainen ML, Linna E, Huikuri HV. Family history and the risk of sudden cardiac death as a manifestation of an acute coronary event. Circulation. 2006; 114:1462-67. [PubMed: 17000909]

58. Kao WH, Arking DE, Post W, Rea TD, Sotoodehnia N, et al. Genetic variations in nitric oxide synthase 1 adaptor protein are associated with sudden cardiac death in US white community-based populations. Circulation. 2009; 119:940-51. [PubMed: 19204306]

59. Lehnart SE, Ackerman MJ, Benson DW Jr, Brugada R, Clancy CE, et al. Inherited arrhythmias: a National Heart, Lung, and Blood Institute and Office of Rare Diseases workshop consensus report about the diagnosis, phenotyping, molecular mechanisms, and therapeutic approaches for primary cardiomyopathies of gene mutations affecting ion channel function. Circulation. 2007; 116:232545. [PubMed: 17998470] 
60. Lehtinen AB, Newton-Cheh C, Ziegler JT, Langefeld CD, Freedman BI, et al. Association of NOS1APgenetic variants with QT interval duration in families from the Diabetes Heart Study. Diabetes. 2008; 57:1108-14. [PubMed: 18235038]

61. Lin DY, Tang ZZ. A general framework for detecting disease associations with rare variants in sequencing studies. Am J Hum Genet. 2011; 89:354-67. [PubMed: 21885029]

62. London B, Michalec M, Mehdi H, Zhu X, Kerchner L, et al. Mutation in glycerol-3-phosphate dehydrogenase 1-like gene (GPD1-L) decreases cardiac $\mathrm{Na}^{+}$current and causes inherited arrhythmias. Circulation. 2007; 116:2260-68. [PubMed: 17967977]

63. Manolio TA, Collins FS, Cox NJ, Goldstein DB, Hindorff LA, et al. Finding the missing heritability of complex diseases. Nature. 2009; 461:747-53. [PubMed: 19812666]

64. Mohamed U, Napolitano C, Priori SG. Molecular and electrophysiological bases of catecholaminergic polymorphic ventricular tachycardia. J Cardiovasc Electrophysiol. 2007; 18:791-97. [PubMed: 17578347]

65. Newton-Cheh C, Eijgelsheim M, Rice KM, de Bakker PI, Yin X, et al. Common variants at ten loci influence QT interval duration in the QTGEN Study. Nat Genet. 2009; 41:399-406. [PubMed: 19305408]

66. Newton-Cheh C, Larson MG, Corey DC, Benjamin EJ, Herbert AG, et al. QT interval is a heritable quantitative trait with evidence of linkage to chromosome 3 in a genome-wide linkage analysis: the Framingham Heart Study. Heart Rhythm. 2005; 2:277-84. [PubMed: 15851319]

67. Nitsch D, Tranchevent LC, Thienpont B, Thorrez L, Van Esch H, et al. Network analysis of differential expression for the identification of disease-causing genes. PLoS ONE. 2009; 4:e5526. [PubMed: 19436755]

68. Noseworthy PA, Havulinna AS, Porthan K, Lahtinen AM, Jula A, et al. Common genetic variants, QT interval, and sudden cardiac death in a Finnish population-based study. Circ Cardiovasc Genet. 2011; 4:305-11. [PubMed: 21511878]

69. Okin PM, Devereux RB, Howard BV, Fabsitz RR, Lee ET, Welty TK. Assessment of QT interval and QT dispersion for prediction of all-cause and cardiovascular mortality in American Indians: the Strong Heart Study. Circulation. 2000; 101:61-66. [PubMed: 10618305]

70. Pfeufer A, Sanna S, Arking DE, Muller M, Gateva V, et al. Common variants at ten loci modulate the QT interval duration in the QTSCD Study. Nat Genet. 2009; 41:407-14. [PubMed: 19305409]

71. Pfeufer A, van Noord C, Marciante KD, Arking DE, Larson MG, et al. Genome-wide association study of PR interval. Nat Genet. 2010; 42:153-59. [PubMed: 20062060]

72. Plant LD, Bowers PN, Liu Q, Morgan T, Zhang T, et al. A common cardiac sodium channel variant associated with sudden infant death in African Americans, SCN5A S1103Y. J Clin Investig. 2006; 116:430-35. [PubMed: 16453024]

73. Post W, Shen H, Damcott C, Arking DE, Kao WH, et al. Associations between genetic variants in the NOS1AP(CAPON) gene and cardiac repolarization in the old order Amish. Hum Hered. 2007; 64:214-19. [PubMed: 17565224]

74. Priori SG, Napolitano C, Schwartz PJ. Low penetrance in the long-QT syndrome: clinical impact. Circulation. 1999; 99:529-33. [PubMed: 9927399]

75. Raitakari OT, Blom-Nyholm J, Koskinen TA, Kahonen M, Viikari JS, Lehtimaki T. Common variation in NOS1AP and KCNH2 genes and QT interval duration in young adults. The Cardiovascular Risk in Young Finns Study. Ann Med. 2008; 41:1-8.

76. Roden DM. Long QT syndrome: reduced repolarization reserve and the genetic link. J Intern Med. 2006; 259:59-69. [PubMed: 16336514]

77. Ruan Y, Liu N, Priori SG. Sodium channel mutations and arrhythmias. Nat Rev Cardiol. 2009; 6:337-48. [PubMed: 19377496]

78. Schouten EG, Dekker JM, Meppelink P, Kok FJ, Vandenbroucke JP, Pool J. QT interval prolongation predicts cardiovascular mortality in an apparently healthy population. Circulation. 1991; 84:1516-23. [PubMed: 1914093]

79. Schwartz PJ, Priori SG, Napolitano C. How really rare are rare diseases? The intriguing case of independent compound mutations in the long QT syndrome. J Cardiovasc Electrophysiol. 2003; 14:1120-21. [PubMed: 14521668] 
80. Sharp DS, Masaki K, Burchfiel CM, Yano K, Schatz IJ. Prolonged $Q T_{\mathrm{c}}$ interval, impaired pulmonary function, and a very lean body mass jointly predict all-cause mortality in elderly men. Ann Epidemiol. 1998; 8:99-106. [PubMed: 9491934]

81. Siscovick DS, Raghunathan TE, Rautaharju P, Psaty BM, Cobb LA, Wagner EH. Clinically silent electrocardiographic abnormalities and risk of primary cardiac arrest among hypertensive patients. Circulation. 1996; 94:1329-33. [PubMed: 8822988]

82. Sotoodehnia N, Isaacs A, de Bakker PI, Dorr M, Newton-Cheh C, et al. Common variants in 22 loci are associated with QRS duration and cardiac ventricular conduction. Nat Genet. 2010; 42:1068-76. [PubMed: 21076409]

83. Splawski I, Timothy KW, Tateyama M, Clancy CE, Malhotra A, et al. Variant of SCN5A sodium channel implicated in risk of cardiac arrhythmia. Science. 2002; 297:1333-36. [PubMed: 12193783]

84. Stecker EC, Sono M, Wallace E, Gunson K, Jui J, Chugh SS. Allelic variants of SCN5A and risk of sudden cardiac arrest in patients with coronary artery disease. Heart Rhythm. 2006; 3:697-700. [PubMed: 16731473]

85. Straus SM, Kors JA, De Bruin ML, van der Hooft CS, Hofman A, et al. Prolonged QTc interval and risk of sudden cardiac death in a population of older adults. J Am Coll Cardiol. 2006; 47:36267. [PubMed: 16412861]

86. Tobin MD, Kahonen M, Braund P, Nieminen T, Hajat C, et al. Gender and effects of a common genetic variant in the NOS1 regulator NOS1AP on cardiac repolarization in 3761 individuals from two independent populations. Int J Epidemiol. 2008; 37:1132-41. [PubMed: 18511491]

87. Tomas M, Napolitano C, De Giuli L, Bloise R, Subirana I, et al. Polymorphisms in the NOS1AP gene modulate QT interval duration and risk of arrhythmias in the long QT syndrome. J Am Coll Cardiol. 2010; 55:2745-52. [PubMed: 20538168]

88. Webster JA, Gibbs JR, Clarke J, Ray M, Zhang W, et al. Genetic control of human brain transcript expression in Alzheimer disease. Am J Hum Genet. 2009; 84:445-58. [PubMed: 19361613]

89. Westenskow P, Splawski I, Timothy KW, Keating MT, Sanguinetti MC. Compound mutations: a common cause of severe long-QT syndrome. Circulation. 2004; 109:1834-41. [PubMed: 15051636]

90. Zheng ZJ, Croft JB, Giles WH, Mensah GA. Sudden cardiac death in the United States, 1989 to 1998. Circulation. 2001; 104:2158-63. [PubMed: 11684624] 


\section{SUMMARY POINTS}

1. Both family studies and the existence of monogenic forms of the disease demonstrate that SCD has a genetic component.

2. Although substantial progress has been made in identifying rare variants underlying monogenic diseases, the relevance of genetic variants in these genes to common forms of SCD remains to be demonstrated.

3. Using endophenotypes, such as QT and QRS intervals, that are themselves risk factors for SCD has led to the identification of genetic variants contributing to common forms of SCD, including variants in NOS1AP and the TKT7 $C A C N A 1 D / P R K C D$ locus. 4. GWAS have identified common variants in the $C X A D R$ and $B A Z 2 B$ loci that modify risk for SCD.

4. Although risk profiling using common variants may not be clinically relevant in the general population, studies in high-risk populations (such as those with LQTS) demonstrate that these variants may be modifiers of the phenotype and help identify those who require intervention.

5. Emerging genetic evidence suggests that SCD may be a final common event representing electrical instability in patients, regardless of their underlying substrate. 


\section{FUTURE ISSUES}

1. What are the specific genes involved in SCD at the associated loci, and how do they alter the risk for the disease?

2. What is the role of rare coding variation in SCD susceptibility?

3. How can we integrate additional genome-scale data (e.g., RNA expression, methylation, and protein expression data) with genetic data to increase our power to identify SCD-associated genes?

4. Can common variants in SCD-associated genes be used clinically to inform therapy in high-risk SCD patient groups? 
a

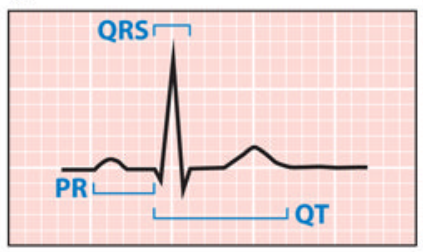

C

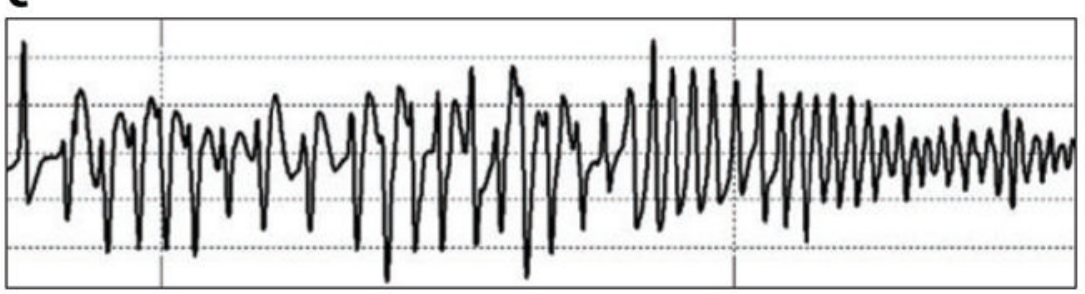

b

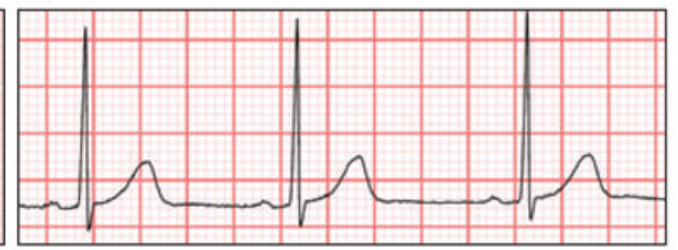

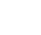

Figure 1.

(a) A normal cardiac electrical cycle. The PR interval represents atrioventricular conduction, the QRS interval represents ventricular depolarization, and the QT interval is a measure of ventricular depolarization (QRS) and repolarization. (b) An electrocardiographic tracing from a patient demonstrating three consecutive normal cardiac electrical cycles (courtesy of Jordan Prutkin). (c) An electrocardiographic tracing from a patient with sudden cardiac death, illustrating ventricular fibrillation (courtesy of the King County Medic One Cardiac Arrest Registry, Seattle, Washington, 2012). 
a

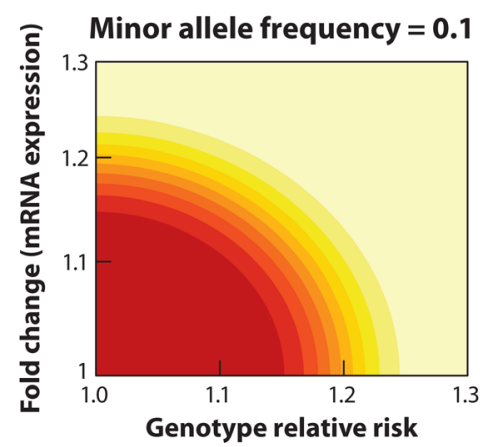

b

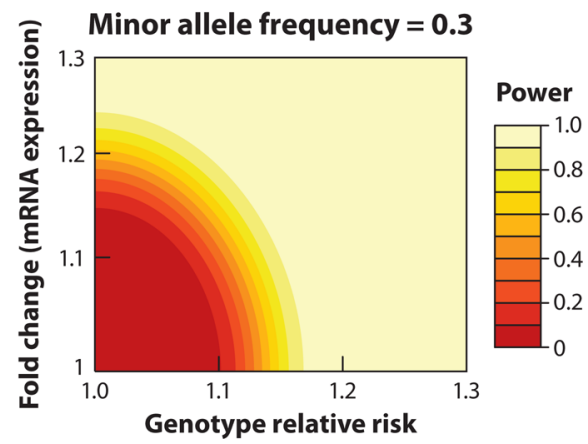

Figure 2.

The power of genome-wide significance $\left(p=5 \times 10^{-8}\right)$ for combined analysis of genomewide association study (GWAS) and mRNA transcript profiling. To illustrate the overall power and highlight the gain in power by incorporating expression data from 250 cases and controls into GWAS analysis, we focus on the analysis of $\sim 5,000$ sudden cardiac death cases and controls. The power gains are quite substantial. (a) For example, for a $10 \%$ minor allele frequency and $0.1 \%$ disease prevalence, using a single-nucleotide polymorphism (SNP)only approach has no power to detect a genotype relative risk of 1.10, whereas a 1.2-fold increase in expression increases power to $80 \%$. (b) For a minor allele frequency of $30 \%$, the power to detect a genotype relative risk of 1.10 is $20 \%$ for a 1.1 -fold change in gene expression, increasing to $>80 \%$ for a 1.2 -fold change. 


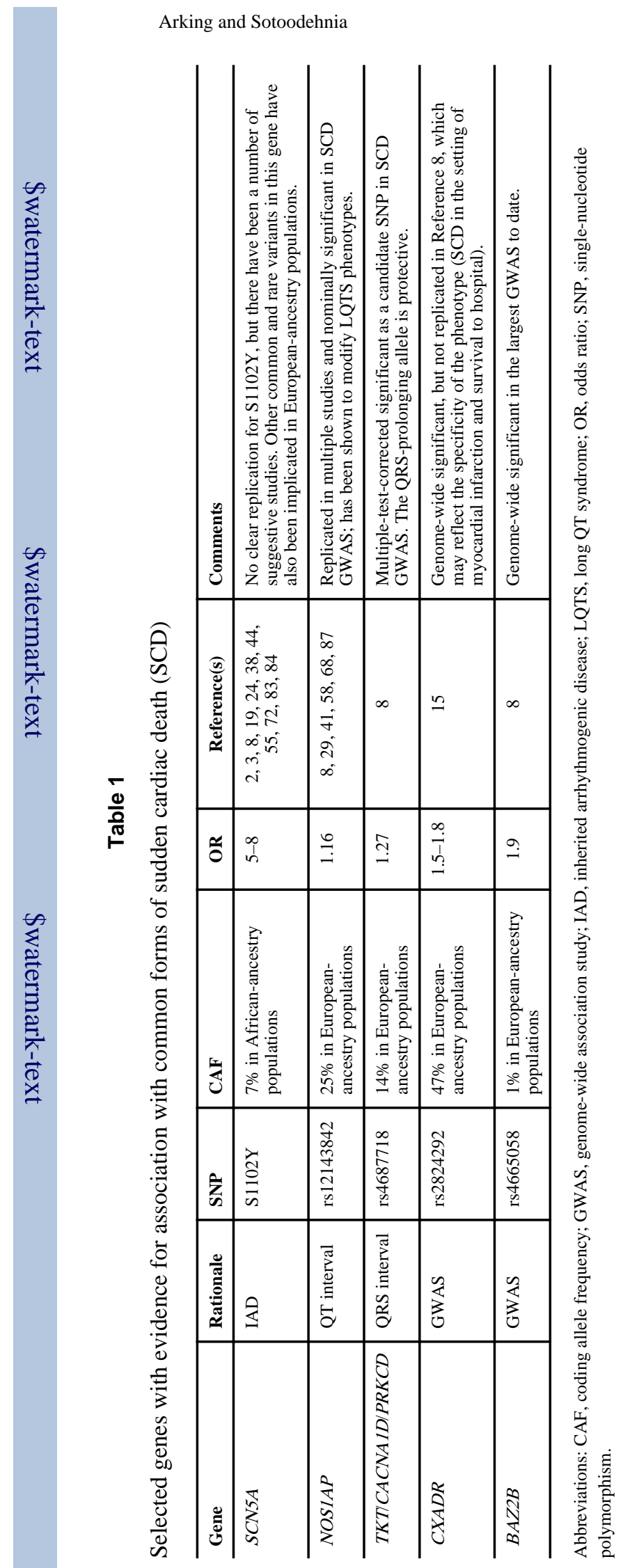

Page 20 